\section{A MINITAB macro for testing marginal homogeneity in contingency tables}

\author{
GLYN M. COLLIS \\ University of Warwick, Coventry, England
}

Matched-pairs designs sometimes produce nominally scaled (categorical) data, as illustrated by the following hypothetical example. Ninety students each take two courses. Each student is given a choice as to how he/she should be assessed on each course. Assessment can be by examination (E), by written work (W), or by oral presentation $(\mathrm{O})$. It is considered useful to know whether the students' choice of assessment procedure differs for the two courses. The data are nominal, comprising three categories, $\mathrm{E}, \mathrm{W}$, and $\mathrm{O}$, which cannot meaningfully be ordered, and a matched-pairs design is used, with each student making a choice for each course.

The resulting data can be tabulated in the conventional form for matched-pairs designs, as in Figure 1, or more conveniently in a square contingency table, as in Figure 2. In the latter format, the question to be asked is whether each row total in Figure 2 is essentially the same as the corresponding column total. This is a question of marginal homogeneity.

Figure 2 can also be viewed as an agreement matrix of the kind for which Cohen's (1960) kappa coefficient could be calculated. In some circumstances, interpretation of kappa may be aided by knowledge of the degree of dissimilarity between the base rates with which the two observers assign items to the various categories: this, too, is a question of marginal homogeneity (Collis, 1985).

Test Statistics. When there are only two categories, McNemar's procedure is appropriate for testing homogeneity of the margins of the resulting $2 \times 2$ table. Stuart (1955) generalized McNemar's test to the case of $k \geq 2$ categories and the resulting $k \times k$ contingency table. Fleiss (1981) gives a useful account of Stuart's test which emphasizes its applicability to matched-pairs designs. Maxwell (1970) illustrates the use of Stuart's test in the analysis of agreement matrices.

A standard notation for contingency tables is used: $n_{i j}$ denotes the frequency in the cell in the $i$ th row and $j$ th column, $n_{i}$ and $n_{. i}$ denote the $i$ th row and column totals respectively, and $n$.. denotes the grand total. $\mathbf{d}$ is a vector of differences $d_{i}=n_{i}-n_{. i}$. Stuart (1955) proposed estimating the elements of $\mathbf{V}$, the covariance matrix of d, as $V_{i i}=n_{i .}+n_{. i}-2 n_{i i}$ and $V_{i j}=-\left(n_{i j}+n_{j i}\right)$. To avoid problems associated with a singular covariance matrix, one $d_{i}$ is dropped from the calculations, together with the corresponding row and column of the covariance matrix.

The author's mailing address is: Department of Psychology, University of Warwick, Coventry CV4 7AL, England.

\begin{tabular}{rcc} 
student & course 1 & course 2 \\
\hline 1 & W & E \\
2 & W & W \\
3 & E & O \\
$:$ & $:$ & $:$ \\
$:$ & $:$ & $:$ \\
90 & O & E \\
\hline W & 20 & 30 \\
O & 30 & 35 \\
\hline$=$ & 90 & 90 \\
\hline
\end{tabular}

Figure 1. Choice of assessment procedure by students taking two courses.

course 2

\begin{tabular}{cc|rrr|r} 
& & $\mathrm{E}$ & $\mathrm{W}$ & $\mathrm{O}$ & total \\
\hline course & $\mathrm{E}$ & 20 & 12 & 8 & 40 \\
1 & $\mathrm{~W}$ & 3 & 15 & 2 & 20 \\
& $\mathrm{O}$ & 2 & 3 & 25 & 30 \\
& total & 25 & 30 & 35 & 90
\end{tabular}

Figure 2. Hypothetical data from a matched-pairs design with nominal data, in the form of a contingency table.

Stuart's statistic is the quadratic form $Q=\mathbf{d}^{\prime} \mathbf{V}^{-1} \mathbf{d} . Q$ is referred to tables of the $\chi^{2}$ distribution with $k-1$ degrees of freedom.

Bhapkar (1966) introduced a test statistic which differs from Stuart's only in that the null case is not assumed in estimating the covariance matrix. Specifically, for Bhapkar's test $V^{*}{ }_{i i}=n_{i .}+n_{. i}-2 n_{i i}-d_{i}^{2} / n_{. .}$and $V^{*}{ }_{i j}=$ 
$-\left(n_{i j}+n_{j i}\right)-d_{i} d_{j} / n_{. .}$. Thus Bhapkar's statistic may be written in terms of Stuart's estimator $\mathbf{V}$ as $Q^{*}=$ $\mathbf{d}^{\prime}\left(\mathbf{V}-\mathbf{n}^{-1} \mathbf{d d}^{\prime}\right)^{-1} \mathbf{d}$. $Q^{*}$ is also referred to the $\chi^{2}$ distribution with $k-1$ degrees of freedom. Unlike Stuart's $Q$, Bhapkar's statistic does not reduce to McNemar's chi square in the $2 \times 2$ case.

When $k \leq 4$, it is possible to calculate Stuart's statistic without resorting to matrix inversion (Fleiss \& Everitt, 1971). For the general case, programs are not difficult to write, provided a matrix inversion routine is available. Dunlap (1984) provides a FORTRAN program for Stuart's test. However, it is very convenient to be able to perform Stuart's and Bhakpar's tests in the environment of the MINITAB package (Ryan, Joiner, \& Ryan, 1982), which is a versatile and friendly package for data analysis and statistics. MINITAB's matrix manipulation commands are well suited to the necessary calculations.

The Macro. Table 1 contains a listing of a MINITAB macro MARGHOMO.MTB. As it stands, this macro will deliver Bhapkar's statistic $Q^{*}$, but it may easily be con-

Table 1

MARGHOMO.MTB: A MINITAB Macro to Test Marginal Homogeneity in a Square Contingency Table

note Stuart/Bhapkar tests of marginal homogeneity
note number of categories in $\mathrm{K} 1$
note first column of contingency table in $\mathrm{CK} 2$
note first column available for workspace : CK3
noecho
let $\mathrm{K} 4=\mathrm{K} 2+\mathrm{K} 1-1$
copy $\mathrm{CK} 2-\mathrm{CK} 4$ into $\mathrm{Ml}$

transpose $\mathrm{MI}$ into $\mathrm{M} 2$

set CK3

$\mathrm{Kl}(1)$

mult M1 by CK3 into M3 row totals

mult M2 by CK3 into M4 \# col totals

add $M 1 \mathrm{M} 2$ into $\mathrm{M} 2$

add M3 M4 into CK3

diag CK3 into M6

subtract M2 from M6 into M6 \# Stuart's covar

subtract M3 from M4 into M5 \# differences

\# convert Stuart's covar to Bhapkar's

transpose M5 into $M 7$

mult $\mathrm{M} 5$ by $\mathrm{M} 7$ into $\mathrm{M} 7$

let $K 4=2 /$ sum(CK3)

mult $\mathrm{K} 4$ by $M 7$ into $M 7$

subtract M7 from M6 into M6 \# Bhapkar's covar

\# eliminate singularity

generate 1 to $\mathrm{K} 1$ into $\mathrm{CK} 3$

let $\mathrm{K} 4=\mathrm{K} 3+\mathrm{K} 1-\mathrm{I}$

indicator CK3 into CK3-CK4

let $\mathrm{K} 4=\mathrm{K} 4-1$

copy $\mathrm{CK} 3-\mathrm{CK} 4$ into $\mathrm{M} 2$

mult M6 by $\mathrm{M} 2$ into M6

transpose $\mathbf{M} 2$ into $\mathbf{M} 2$

mult $\mathrm{M} 2$ by $\mathrm{M} 6$ into $\mathrm{M} 6$

mult M2 by M5 into M5

\# calculate quadratic form

invert $\mathrm{M} 6$ into $\mathrm{M} 7$

mult $\mathrm{M} 7$ by $\mathrm{M} 5$ into $\mathrm{M} 2$

transpose M5 into M5

mult M5 by M2 into M2

note the final ANSWER is chisquare

note with $K 1-1$ degrees of freedom verted to deliver Stuart's $Q$ by deleting the five commands in Lines 18-23 of the listing.

Before running the macro, it is necessary to assign values to the three MINITAB constants $\mathrm{K} 1, \mathrm{~K} 2$, and $\mathrm{K} 3$. $\mathrm{K} 1$ is set to $k$, the number of categories. $\mathrm{K} 2$ points to the first of a set of $k$ consecutive columns that contain the data in the form of a $k \times k$ contingency table, and $\mathrm{K} 3$ points to the first of $k$ columns that can be used for workspace (these will be overwritten when the macro is run). Thus, if $k=3$ and the $3 \times 3$ contingency table is stored in Columns $\mathrm{C} 8-\mathrm{C} 10, \mathrm{~K} 1$ should be set to 3 , and $\mathrm{K} 2$ should be set to 8. Assuming Columns C21-C23 do not contain data that the user requires, $\mathrm{K} 3$ may be set to 21 .

The macro is run using the command EXEC 'MARGHOMO'. The MINITAB Constant K4 and Matrices $\mathrm{M} 1-\mathrm{M} 7$ are used by the macro, and any prior contents will be lost. At the termination of the macro run, M1 contains the contingency table, M2 the $Q^{*}$ (or $Q$ ) statistic, M3 the row totals, M4 the column totals, M5 the first $k-1 d_{i} \mathrm{~s}$, M6 the corresponding covariance matrix, and $M 7$ its inverse. MINITAB prints out ANSWER = followed by the value of the $Q^{*}$ statistic (or $Q$ ). (For the special case of a $2 \times 2$ table, MINITAB prints out more than one ANSWER. This is because MINITAB automatically does this whenever a matrix multiplication yields a scalar product, which happens for intermediate steps in the $2 \times 2$ case. The last ANSWER is the $Q^{*}$ or $Q$ statistic.) For the data given in Figure 2, $Q^{*}=$ 10.1124 .

The macro requires MINITAB Release 82.1 or later. It normally is possible to analyze quite large contingency tables, limited only by the size of matrices allowed, and the number of columns that may be copied with one COPY command, in a particular implementation of MINITAB.

\section{REFERENCES}

BHAPKaR, V. P. (1966). A note on the equivalence of two test criteria for hypotheses in categorical data. Journal of the American Statistical Association, 61, 228-235.

CoHEN, J. (1960). A coefficient of agreement for nominal scales. Educational \& Psychological Measurement, 20, 37-46.

Colus, G. M. (1985). Kappa, measures of marginal symmetry and intraclass correlations. Educational \& Psychological Measurement, 45, 45-62.

DUNLAP, W. P. (1984). A FORTRAN IV program for the StuartMaxwell test. Behavior Research Methods, Instruments, \& Computers, 16, 489-491.

FLeiss, J. L. (1981). Statistical Methods for rates and proportions (2nd ed.). New York: Wiley.

Fleiss, J. L., \& EveritT, B. S. (1971). Comparing the marginal totals of square contingency tables. British Journal of Mathematical \& Statistical Psychology, 24, 117-123.

MAXWELL, A. E. (1970). Comparing the classification of subjects by two independent judges. British Journal of Psychiatry, 116, 651-655.

RYAN, T. A., JOINER, B. L., \& RYAN, B. F. (1982). MINITAB reference manual. MINITAB project, Pennsylvania State University, University Park, PA

STUART, A. (1955). A test for homogeneity of the marginal distributions in a two-way classification. Biometrika, 42, 412-416.

(Revision received for publication February 4, 1986.) 